\title{
An exact solution for the KPZ equation with flat initial conditions
}

\author{
Pasquale Calabrese ${ }^{1}$ and Pierre Le Doussal ${ }^{2}$ \\ ${ }^{1}$ Dipartimento di Fisica dell'Università di Pisa and INFN, 56127 Pisa Italy \\ ${ }^{2}$ CNRS-Laboratoire de Physique Théorique de l'Ecole Normale Supérieure, 24 rue Lhomond, 75231 Paris Cedex, France
}

(Dated: October 22, 2018)

\begin{abstract}
We provide the first exact calculation of the height distribution at arbitrary time $t$ of the continuum KPZ growth equation in one dimension with flat initial conditions. We use the mapping onto a directed polymer (DP) with one end fixed, one free, and the Bethe Ansatz for the replicated attractive boson model. We obtain the generating function of the moments of the DP partition sum as a Fredholm Pfaffian. Our formula, valid for all times, exhibits convergence of the free energy (i.e. KPZ height) distribution to the GOE Tracy Widom distribution at large time.
\end{abstract}

PACS numbers:

The continuum Kardar-Parisi-Zhang (KPZ) equation is the simplest equation describing the non-equilibrium growth in time $t$ of an interface of height $h(x, t)$ in presence of noise [1]. In one dimension, i.e. $x \in R$, it reads

$$
\partial_{t} h=\nu \nabla^{2} h+\frac{1}{2} \lambda_{0}(\nabla h)^{2}+\eta(x, t),
$$

where $\overline{\eta(x, t) \eta\left(x, t^{\prime}\right)}=D \delta\left(x-x^{\prime}\right) \delta\left(t-t^{\prime}\right)$ is a centered Gaussian white noise. Originally conceived to describe growth by random deposition and diffusion, it defines a universality class believed to encompass an astounding variety of models and physical systems [2]. The growing KPZ interface becomes, at large $t$, statistically self-affine with universal scaling exponents. In $d=1$, its width is predicted [3] to grow as $\delta h \sim t^{1 / 3}$, as observed in experiments [4, 5]. The KPZ problem also maps to forced Burgers turbulence [6], and to the equilibrium statistical mechanics of a directed polymer (DP) in a random potential, the simplest example of a glass [7] with applications to vortex lines [8], domain walls [9], and biophysics [10]. However, despite its importance and universality, the KPZ equation has vigorously resisted analytical solutions.

Progress in analytical understanding of the KPZ class in $d=1$ came from exact solutions of a lattice DP model at zero temperature [11], discrete growth models such as the PNG model [12, 13], asymmetric exclusion models [14] and vicious walkers 15]. An analogous to the height field $h(x, t)$ was identified and, in the large size limit, its one-point (scaled) probability distribution was shown to equal the (scaled) distribution of the smallest eigenvalue of a random matrix drawn from the famous Gaussian ensembles, the so-called Tracy Widom (TW) distribution 16], which appears in many other contexts 17. It was found [12, 18] that one gets either the TW distribution $F_{2}(s)$ of the Gaussian unitary ensemble (GUE) or $F_{1}(s)$ of the Gaussian orthogonal ensemble (GOE) for droplet and flat initial conditions respectively. The corresponding many point distributions were identified as determinantal space-time processes, the Airy process, $A i_{2}$ for droplet, and $A i_{1}$ for flat naturally expressed with the use of Fredholm determinants [19].

These advances gave valuable, but only indirect information on the continuum KPZ equation, i.e. a conjecture for its infinite $t$ limit (termed the KPZ renormalization fixed point [20]). Only recently we 21], and other workers 22 25], were able to directly solve the continuum problem and, until now, only within the droplet initial condition. In addition to the convergence to $F_{2}(s)$ at large $t$, the unveiled remarkable feature is that a proper generating function $g(s)$ (recalled below) remains a Fredholm determinant for all times $t$, hence leading to an exact solution for the universal crossover in time in the continuum KPZ equation. This universal distribution (depending on a single parameter $t$ ) describes in the DP framework the high temperature regime 21] that has remarkable universal features [26]. In the growth problem, this corresponds to a universal large diffusivity-weak noise limit, at fixed correlation length of the noise.

In this Letter we obtain the corresponding exact result for the continuum KPZ equation for the case of flat initial conditions, most often encountered in experiments [5]. We obtain here the generating function of the integer moments of the DP partition sum $Z \equiv e^{\frac{\lambda_{0}}{2 \nu} h}$ (see below)

$$
g_{\lambda}(s)=\sum_{n=0}^{\infty} \frac{\left(-e^{-\lambda s}\right)^{n}}{n !} \overline{Z^{n}} \quad, \quad \lambda=\frac{1}{2}\left(\bar{c}^{2} t / T^{5}\right)^{1 / 3}
$$

with $\bar{c}=D \lambda_{0}^{2}, T=2 \nu$, as a Fredholm Pfaffian for any time $t$. The DP free energy, and the height field at a given point, take the form

$$
\frac{\lambda_{0}}{2 \nu} h \equiv \ln Z=v_{0} t+\lambda \xi_{t},
$$

where $g_{\lambda}(s)=\overline{\exp \left(-e^{\lambda\left(\xi_{t}-s\right)}\right)}$. In the large time limit $g_{\infty}(s) \equiv \lim _{\lambda \rightarrow \infty} g_{\lambda}(s)=\operatorname{Prob}\left(\xi_{t}<s\right)$ and we find that the distribution variable $\xi_{t}$ converge to the GOE Tracy Widom distribution $\operatorname{Prob}\left(\xi_{t}<s\right)=F_{1}(s)$. As in 21] we use the Bethe Ansatz (BA) for the replicated boson model with $\delta$ attraction, and sum over all excited states, treating now the case of a DP with one end fixed and the other free. The calculation is far more complicated 
than [21] as we need the spatial integrals of the Bethe wavefunctions. Technically, this is surmounted by solving a half-space model in the proper limit.

The solution of (1) for a given initial condition can be written, using the Cole-Hopf transformation $Z \equiv e^{\frac{\lambda_{0}}{2 \nu} h}$

$$
e^{\frac{\lambda_{0}}{2 \nu} h(x, t)}=\int d y Z(x, t \mid y, 0) e^{\frac{\lambda_{0}}{2 \nu} h(y, t=0)},
$$

in terms of the partition function of a DP, at temperature $T=2 \nu$ and in the random potential $V(x, t)=\lambda_{0} \eta(x, t)$, i.e the sum over paths $x(\tau) \in R$ starting at $x(0)=y$ and ending at $x(t)=x$

$$
Z(x, t \mid y, 0)=\int_{x(0)=y}^{x(t)=x} D x(\tau) e^{-\frac{1}{T} \int_{0}^{t} d \tau\left[\frac{1}{2}\left(\frac{d x}{d \tau}\right)^{2}+V(x(\tau), \tau)\right]},
$$

with initial condition $Z(x, t=0 \mid y, 0)=\delta(x-y)$. In this continuum model the disorder correlation length is zero, i.e. $\overline{V(x, t) V\left(x^{\prime}, t\right)}=\bar{c} \delta\left(t-t^{\prime}\right) \delta\left(x-x^{\prime}\right)$ with $\bar{c}=D \lambda_{0}^{2}$, hence one can perform a rescaling $x \rightarrow T^{3} x / \bar{c}$ and $t \rightarrow$ $2 T^{5} t / \bar{c}^{2}$, and work in units such that $T=1$ and $\bar{c}=$ 1 , as we do below. The continuum model (5) describes the high $T$ limit of the DP on a lattice as discussed in [21, 26]. For the KPZ interface this continuum model describes the universal limit where the characteristic time $t^{*}=2(2 \nu)^{5} / D^{2} \lambda_{0}^{4}$ and space $x^{*}=\sqrt{\nu t^{*}}$ scales are much larger than the correlation lengths of the noise.

In 21] we obtained the distribution of $\ln Z$ for a DP with the two ends fixed $Z=Z(0, t \mid 0,0)$. From (4) this corresponds to a wedge initial condition for the KPZ interface, $\frac{\lambda_{0}}{2 \nu} h_{\text {wedge }}(x, t=0)=-w|x|$, in the limit of a narrow wedge $w \rightarrow \infty$. Here we solve the flat interface initial conditions for KPZ, i.e. $h(x, t=0)=0$, hence the opposite limit $w \rightarrow 0^{+}$of the wedge. It corresponds to the DP with one end fixed, one end free, i.e. $Z_{\text {flat }}(x, t)=\int d y Z(x, t \mid y, 0)$. To achieve that we found it easier to study the (left) half-space problem

$$
Z_{w}(x, t)=\int_{-\infty}^{0} d y e^{w y} Z(x, t \mid y, 0)
$$

with $Z(x, t=0)=\theta(-x) e^{w x}$. It is easy to show 31 that at $w=0^{+}$the half space model interpolates between (i) the narrow wedge initial condition for $x \rightarrow+\infty$, since the polymer is stretched and (ii) the flat (full-space) initial condition for $x \rightarrow-\infty$, hence we study below $\lim _{x \rightarrow-\infty} \lim _{w \rightarrow 0} Z_{w}(x, t)=Z_{\text {flat }}(x, t)$.

As well known [27] the calculation of the $n$-th integer moment of a DP partition sum can be expressed as a quantum mechanical problem for $n$ particles described by the (attractive) Lieb-Liniger Hamiltonian [28]

$$
H_{n}=-\sum_{j=1}^{n} \frac{\partial^{2}}{\partial x_{j}^{2}}-2 \bar{c} \sum_{1 \leq i<j \leq n} \delta\left(x_{i}-x_{j}\right)
$$

Generalizing [21], the quantum mechanical expectation for $\overline{Z_{w}(x, t)^{n}}$ is written as a sum over the un-normalized eigenfunctions $\Psi_{\mu}$ (of norm denoted $\|\mu\|$ ) of $H_{n}$ with energies $E_{\mu}$ [33]:

$$
\begin{aligned}
& \overline{Z_{w}(x, t)^{n}}=\int_{y_{i}<0} e^{w \sum_{i=1}^{n} y_{i}}\left\langle y_{1} \ldots y_{n}\left|e^{-t H_{n}}\right| x \ldots x\right\rangle \\
& =\sum_{\mu} \Psi_{\mu}^{*}(x, . . x) \int^{w} \Psi_{\mu} \frac{1}{\|\mu\|^{2}} e^{-t E_{\mu}} \\
& \int^{w} \Psi_{\mu}:=\int_{y_{i}<0} e^{w \sum_{i=1}^{n} y_{i}} \Psi_{\mu}\left(y_{1}, . . y_{n}\right)
\end{aligned}
$$

where we used the fact that only symmetric (i.e. bosonic) eigenstates contribute. The Bethe states $\Psi_{\mu}$ are superpositions of plane waves [28] over all permutations $P$ of the rapidities $\lambda_{j}(j=1, . . n)$ and we use the convention

$$
\Psi_{\mu}\left(x_{1}, . . x_{n}\right)=\sum_{P} A_{P} \prod_{j=1}^{n} e^{i \lambda_{P_{\ell}} x_{\ell}}
$$

where the coefficients $A_{P}=\prod_{n \geq \ell>k \geq 1}\left(1+\frac{\left.i \bar{c} \operatorname{sgn}\left(x_{\ell}-x_{k}\right)\right)}{\lambda_{P_{\ell}}-\lambda_{P_{k}}}\right)$. The general eigenstates are built by partitioning the $n$ particles into a set of $n_{s}$ bound-states formed by $m_{j} \geq 1$ particles with $n=\sum_{j=1}^{n_{s}} m_{j}$. Because we work with $w=0^{+}$, we can take directly the system size $L=\infty$ and in that limit 29] each bound state is a perfect string, i.e. a set of rapidities $\lambda^{j, a}=k_{j}+\frac{i \bar{c}}{2}\left(m_{j}+1-2 a\right)$, where $a=1, \ldots, m_{j}$ labels the rapidities within the string. Such eigenstates have momentum $K_{\mu}=\sum_{j=1}^{n_{s}} m_{j} k_{j}$ and energy $E_{\mu}=\sum_{j=1}^{n_{s}}\left(m_{j} k_{j}^{2}-\frac{\bar{c}^{2}}{12} m_{j}\left(m_{j}^{2}-1\right)\right)$. The groundstate corresponds to a single $n$-string with $k_{1}=0$.

In (8) one already knows $\Psi_{\mu}^{*}(x, . . x)=n ! e^{-i x \sum_{\alpha} \lambda_{\alpha}}$ and the norms $\|\mu\|$ of the string states 30]

$$
\begin{aligned}
& \|\mu\|^{-2}=\frac{(\bar{c})^{n}}{n !(L \bar{c})^{n_{s}}} \prod_{j=1}^{n_{s}} m_{j}^{-2} \prod_{1 \leq i<j \leq n_{s}} \Phi_{k_{i}, m_{i}, k_{j}, m_{j}} \\
& \Phi_{k_{i}, m_{i}, k_{j}, m_{j}}:=\frac{\left(k_{i}-k_{j}\right)^{2}+\left(m_{i}-m_{j}\right)^{2} \bar{c}^{2} / 4}{\left(k_{i}-k_{j}\right)^{2}+\left(m_{i}+m_{j}\right)^{2} \bar{c}^{2} / 4}
\end{aligned}
$$

The new difficulty, i.e. computing the spatial integral of the Bethe states, simplifies dramatically for the halfspace model. Using the symmetry of Eq. (10), we have

$$
\begin{aligned}
\int^{w} \Psi_{\mu} & =n ! \sum_{P} G_{\lambda_{P_{1}}, \ldots \lambda_{P_{n}}}^{w} \prod_{n \geq \ell>k \geq 1}\left(1+\frac{i \bar{c}}{\lambda_{P_{\ell}}-\lambda_{P_{k}}}\right) \\
G_{\lambda_{1}, . . \lambda_{n}}^{w} & =\prod_{j=1}^{n} \frac{1}{j w+i \lambda_{1}+. .+i \lambda_{j}}
\end{aligned}
$$

From the remarkable properties of the BA, it can be reexpressed, for any $n$ and set of rapidities (with $\bar{c}=1$ ):

$$
\int^{w} \Psi_{\mu}=\frac{n !}{i^{n} \prod_{\alpha=1}^{n}\left(\lambda_{\alpha}-i w\right)} \prod_{1 \leq \alpha<\beta \leq n} \frac{i+\lambda_{\alpha}+\lambda_{\beta}-2 i w}{\lambda_{\alpha}+\lambda_{\beta}-2 i w} .
$$


If we now inject the string solution $\lambda_{j, a}=\frac{i}{2}\left(m_{j}+1-\right.$ $2 a)+k_{j}$, we find after some elementary manipulations

$$
\begin{aligned}
& \int^{w} \Psi_{\mu}=n !(-2)^{n} \prod_{i=1}^{n_{s}} S_{m_{i}, k_{i}}^{w} \prod_{1 \leq i<j \leq n_{s}} D_{m_{i}, k_{i}, m_{j}, k_{j}}^{w}, \\
& S_{m_{i}, k_{i}}^{w}=\frac{\Gamma\left(\kappa_{i i}-m_{i}\right)}{\Gamma\left(\kappa_{i i}\right)}, \\
& D_{m_{i}, k_{i}, m_{j}, k_{j}}^{w}=\frac{\Gamma\left(\kappa_{i j}-\frac{m_{i}+m_{j}}{2}\right) \Gamma\left(\kappa_{i j}+\frac{m_{i}+m_{j}}{2}\right)}{\Gamma\left(\kappa_{i j}+\frac{m_{i}-m_{j}}{2}\right) \Gamma\left(\kappa_{i j}-\frac{m_{i}-m_{j}}{2}\right)},
\end{aligned}
$$

$$
Z\left(n_{s}, x\right)=\sum_{m_{1}, \ldots m_{n_{s}}=1}^{\infty} \prod_{j=1}^{n_{s}}\left[\frac{2^{m_{j}}}{m_{j}} \int_{k_{j}} S_{m_{j}, k_{j}}^{w} e^{\left(m_{j}^{3}-m_{j}\right) \frac{t}{12}-m_{j} k_{j}^{2} t-\lambda m_{j} s-i x m_{j} k_{j}}\right] \prod_{1 \leq i<j \leq n_{s}} D_{m_{i}, k_{i}, m_{j}, k_{j}}^{w} \Phi_{k_{i}, m_{i}, k_{j}, m_{j}} .
$$

Upon inspection we find that the limit of interest, $w=0^{+}$, is dominated by poles in the $k_{i}$ integrations, with $S_{m_{i}, k_{i}}^{w} \sim \frac{(-1)^{m_{i}}}{\Gamma\left(m_{i}\right)\left(2 i k_{i}+2 w\right)}$ and $D_{m_{i}, k_{i}, m_{j}, k_{j}}^{w} \sim$ $\frac{(-1)^{m_{i}} m_{i}}{i\left(k_{i}+k_{j}\right)+2 w} \delta_{m_{i}, m_{j}}$, and that the regular parts do not contribute. Replacing $1 /\left(i k+0^{+}\right) \rightarrow \pi \delta(k)$ yields an $x$ independent result, which can be shown to equal the limit $x \rightarrow-\infty$, i.e. the flat initial condition for KPZ, on which with $\kappa_{i j}=-i k_{i}-i k_{j}-2 w+1$.

We have now all ingredients to compute the generating function (2) with $Z \equiv Z_{w}(x, t)$. Writing the sum over states in (8) as all partitioning of $n$ particles into $n_{s}$ strings and using that for $L \rightarrow \infty$ the string momenta $m_{j} k_{j}$ correspond to free particles [30] (i.e. $\sum_{k_{j}} \rightarrow$ $\left.m_{j} L \int \frac{d k_{j}}{2 \pi} \equiv m_{j} L \int_{k_{j}}\right)$, Eq. (22) becomes a sum over string configurations $g_{\lambda}(s)=1+\sum_{n_{s}=1}^{\infty} \frac{1}{n_{s} !} Z\left(n_{s}, s\right)$ with

$$
\begin{aligned}
& Z\left(n_{s}\right)=\sum_{m_{i} \geq 1} \prod_{j=1}^{n_{s}} \int_{k_{j}} \prod_{q=1}^{m_{j}} \frac{-2}{2 i k_{j}+q} e^{\frac{\lambda^{3}}{3} m_{j}^{3}-4 m_{j} k_{j}^{2} \lambda^{3}-\lambda m_{j} s} \\
& \times \operatorname{Pf}\left[\left(\begin{array}{cc}
\frac{2 \pi}{2 i k_{i}} \delta\left(k_{i}+k_{j}\right)(-1)^{m_{i}} \delta_{m_{i}, m_{j}}+\frac{1}{4}(2 \pi)^{2} \delta\left(k_{i}\right) \delta\left(k_{j}\right)(-1)^{\min \left(m_{i}, m_{j}\right)} \operatorname{sgn}\left(m_{i}-m_{j}\right) & \frac{1}{2}(2 \pi) \delta\left(k_{i}\right) \\
-\frac{1}{2}(2 \pi) \delta\left(k_{j}\right) & \frac{2 i k_{i}+m_{i}-2 i k_{j}-m_{j}}{2 i k_{i}+m_{i}+2 i k_{j}+m_{j}}
\end{array}\right)\right]_{2 n_{s} \times 2 n_{s}} .
\end{aligned}
$$

We recall that for an antisymmetric matrix $A$ of size $2 n_{s}$

$$
\operatorname{Pf} A=\sum_{\sigma \in S_{2 n_{s}}, \sigma(2 j-1)<\sigma(2 j)}(-1)^{\sigma} \prod_{i=1}^{n_{s}} A_{\sigma(2 i-1), \sigma(2 i)},
$$

with $(\operatorname{Pf} A)^{2}=\operatorname{det} A$. We can now use the Airy trick [21, 22] $\prod_{j} e^{\frac{1}{3} \lambda^{3} m_{j}^{3}}=\prod_{j} \int_{y_{j}} A i\left(y_{j}\right) e^{\lambda y_{j}}$ and decouple the denominators in the lower right corner using auxiliary integrals $\prod_{j} \int_{v_{j}>0} e^{-v_{j} A_{j}}=\prod_{j} \frac{1}{A_{j}}$ and the numerators using derivatives. We perform rescaling $k_{j} \rightarrow k_{j} / \lambda$, and shifts $y_{j} \rightarrow y_{j}+v_{j}-4 k_{j}^{2}+s$. The summations over the $m_{i}$ can be performed exactly inside the Pfaffian and we we now focus. The result is the sum of the residues associated to configurations where the $n_{s}=2 N+M$ strings split into $N$ pairs of strings of opposite momenta with same particle number $m$ and $M$ single strings of zero momentum with all distinct number of particles. After some non-trivial manipulations, detailed in [31], we bring the result in the form of a Pfaffian: arrive at our main result for $g_{\lambda}(s)$ as a Fredholm Pfaffian

$$
\begin{aligned}
& g_{\lambda}(s)=\operatorname{Pf}[\mathbf{J}+\mathbf{K}]=\sum_{n_{s}=0}^{\infty} \frac{1}{n_{s} !} Z\left(n_{s}\right), \\
& Z\left(n_{s}\right)=\prod_{j=1}^{n_{s}} \int_{v_{j}>0} \operatorname{Pf}\left[\mathbf{K}\left(v_{i}, v_{j}\right)\right]_{2 n_{s}, 2 n_{s}},
\end{aligned}
$$

where $\mathbf{J}=\left(\begin{array}{cc}0 & I \\ -I & 0\end{array}\right)$ and $\mathbf{K}$ is an antisymmetric 2 by 2 matrix kernel of components $K_{a b} \equiv K_{a b}\left(v_{i}, v_{j}\right)$ with

$$
K_{11}=\int_{y_{1}, y_{2}, k} A i\left(y_{1}+v_{i}+s+4 k^{2}\right) A i\left(y_{2}+v_{j}+s+4 k^{2}\right)\left[\frac{e^{-2 i\left(v_{i}-v_{j}\right) k}}{2 i k} f_{k / \lambda}\left(e^{\lambda\left(y_{1}+y_{2}\right)}\right)+\frac{\pi \delta(k)}{2} F\left(2 e^{\lambda y_{1}}, 2 e^{\lambda y_{2}}\right)\right],
$$




$$
\begin{aligned}
K_{12} & =\frac{1}{2} \int_{y} A i\left(y+s+v_{i}\right)\left(e^{-2 e^{\lambda y}}-1\right) \delta\left(v_{j}\right), \\
K_{22} & =2 \delta^{\prime}\left(v_{i}-v_{j}\right)
\end{aligned}
$$

and the functions

$$
\begin{aligned}
& f_{k}(z)=\frac{-2 \pi k z_{1} F_{2}(1 ; 2-2 i k, 2+2 i k ;-z)}{\sinh (2 \pi k) \Gamma(2-2 i k) \Gamma(2+2 i k)}, \\
& F\left(z_{i}, z_{j}\right)=\sinh \left(z_{2}-z_{1}\right)+e^{-z_{2}}-e^{-z_{1}}+\int_{0}^{1} d u \\
& \times J_{0}\left(2 \sqrt{z_{1} z_{2}(1-u)}\right)\left[z_{1} \sinh \left(z_{1} u\right)-z_{2} \sinh \left(z_{2} u\right)\right] .
\end{aligned}
$$

The full analysis of this result is performed in [31]. Here, we first point out the simple one-string contribution $\left(n_{s}=1\right), Z(1)=\int_{v>0} K_{12}(v, v)$ leading to

$$
Z(1)=\frac{1}{2} \int d y\left(e^{-2 e^{\lambda y}}-1\right) A i(y+s),
$$

also obtained 31] from the ground state for each $n$, which gives the leading asymptotics of $g_{\lambda}(s)$ for large $s>0$. Using $\operatorname{det}\left[\begin{array}{ll}A & B \\ C & D\end{array}\right]=\operatorname{det} D \operatorname{det}\left[A-B D^{-1} C\right]$, $g_{\lambda}^{2}(s)=\operatorname{Det}[\mathbf{I}-\mathbf{J K}]$ can be written (for any time) in a form suitable for numerical evaluation [31]. The Pfaffian reported above allows simpler analytic manipulations.

In the large time (large $\lambda$ ) limit, one already sees from (20) that $Z(1) \rightarrow-\int_{y>0} A i(2 y+s)=-\operatorname{Tr} \mathcal{B}_{s}$ where $\mathcal{B}_{s}=\theta(x) A i(x+y+s) \theta(y)$ is the GOE kernel, as shown by Ferrari and Spohn 32 . This extends to all $n_{s}$ i.e. we find that

$$
\lim _{\lambda \rightarrow+\infty} Z\left(n_{s}\right)=(-1)^{n_{s}} \int_{x_{1}, \ldots x_{n_{s}}} \operatorname{det}\left[\mathcal{B}_{s}\left(x_{i}, x_{j}\right)\right]_{n_{s} \times n_{s}}
$$

Hence $g_{\infty}(s)=F_{1}(s)=\operatorname{det}\left[I-\mathcal{B}_{s}\right]$ the Fredholm determinant expression for the GOE Tracy Widom distribution. This is obtained using $\lim _{\lambda \rightarrow+\infty} f_{k / \lambda}\left(e^{\lambda y}\right)=-\theta(y)$ and $\lim _{\lambda \rightarrow+\infty} F\left(2 e^{\lambda y_{1}}, 2 e^{\lambda y_{2}}\right)=\theta\left(y_{1}+y_{2}\right)\left(\theta\left(y_{1}\right) \theta\left(-y_{2}\right)-\right.$ $\left.\theta\left(y_{2}\right) \theta\left(-y_{1}\right)\right)$. We checked (21) explicitly up to $n_{s}=4$, but we report the proof in [31]. For $n_{s}$ even it follows from a slight generalization of [32], namely $\operatorname{det}(I \mp$ $\left.\mathcal{B}_{s}\right) / \operatorname{det}\left(I \pm \mathcal{B}_{s}\right)=\int_{x>0}\left(I \pm \mathcal{B}_{s}\right)^{-1}(x, 0)$.

To summarize we have obtained the generating function for the distribution of the free energy of the DP with one free end, i.e. of the height of the continuum KPZ interface, for arbitrary time. At large time the distribution crosses over to the GOE Tracy Widom distribution $F_{1}(s)$. Further properties of the finite time, including extracting $P(f)$ and numerics are studied in 31].

We thank A. Rosso for discussions and for helpful numerical checks [31] of (i) low integer moments of $Z$ at small $t$ (ii) the variance of $\ln Z$ at large $t$. PC thanks LPTENS, and PLD thanks KITP for hospitality. This work was supported by ANR grant 09-BLAN-0097-01/2.
[2] A.-L. Barabasi, H.E. Stanley, Fractal concepts in surface growth, Cambridge University Press (1995); J. Krug, Adv. Phys. 46, 139 (1997).

[3] D.A. Huse, C.L. Henley, and D.S. Fisher, Phys. Rev. Lett. 55, 2924 (1985).

[4] W.M. Tong and R.W. Williams, Ann. Rev. Phys. Chem. 45, 401 (1994). M. Degawa et al, Phys. Rev. Lett. 97, 080601 (2006). K. Takeuchi and M. Sano, Phys. Rev. Lett. 104, 230601 (2010).

[5] L. Miettinen et al., Eur. Phys. J. B 46, 55 (2005).

[6] J. Bec and K. Khanin, Phys. Rep. 447, 1 (2007).

[7] M. Kardar and Y-C. Zhang, Phys. Rev. Lett. 58, 2087 (1987); T. Halpin-Healy and Y-C. Zhang, Phys. Rep. 254, 215 (1995).

[8] G. Blatter et al., Rev. Mod. Phys. 66, 1125 (1994).

[9] S. Lemerle et al., Phys. Rev. Lett. 80, 849 (1998).

[10] T. Hwa and M. Lassig, Phys. Rev. Lett. 76, 2591 (1996).

[11] K. Johansson, Comm. Math. Phys. 209, 437 (2000).

[12] M. Prahofer and H. Spohn, Phys. Rev. Lett. 84, 4882 (2000); J. Stat. Phys. 108, 1071 (2002); 115, 255 (2004).

[13] J. Baik and E.M. Rains, J. Stat. Phys. 100, 523 (2000).

[14] P.L. Ferrari and H. Spohn, Comm. Math. Phys. 265, 1 (2006); J. de Gier and F. H. L. Essler, arXiv:1101.3235.

[15] J. Rambeau and G. Schehr, 1102.1640; P. Forrester, S. N. Majumdar, G. Schehr, Nucl. Phys. B 844, 500 (2011).

[16] C.A. Tracy and H. Widom, Comm. Math. Phys. 159, 151 (1994).

[17] J. Baik, P.A. Deift and K. Johansson, J. Amer. Math. Soc. 12, 1119 (1999); S.N. Majumdar and S. Nechaev, Phys. Rev. E 72, 020901 (2005); 69, 011103 (2004);

[18] P.L. Ferrari, Comm. Math. Phys. 252, 77 (2004); T. Sasamoto, J. Phys. A 38, L549 (2005).

[19] P. L. Ferrari and H. Spohn, arXiv:1003.0881

[20] I. Corwin and J. Quastel, arXiv:1103.3422

[21] P. Calabrese, P. Le Doussal and A. Rosso, EPL 90, 20002 (2010).

[22] V. Dotsenko, EPL 90, 20003 (2010); J. Stat. Mech. P07010 (2010); V. Dotsenko and B. Klumov, ibid. (2010) P03022.

[23] T. Sasamoto and H. Spohn, Phys. Rev. Lett. 104, 230602 (2010); Nucl. Phys. B 834, 523 (2010); J. Stat. Phys. 140, 209 (2010).

[24] G. Amir, I. Corwin, J. Quastel, Comm. Pure Appl. Math 64, 466 (2011).

[25] S. Prolhac and H. Spohn, J. Stat. Mech. (2011) P03020; (2011) P01031.

[26] S. Bustingorry, P. Le Doussal and A. Rosso Phys. Rev. B 82, 140201 (2010).

[27] M. Kardar, Nucl. Phys. B 290, 582 (1987); E. Brunet and B. Derrida, Phys. Rev. E 61, 6789 (2000); Physica A 279, 395 (2000).

[28] E. H. Lieb and W. Liniger, Phys. Rev. 130, 1605 (1963).

[29] J. B. McGuire, J. Math. Phys. 5, 622 (1964).

[30] P. Calabrese and J.-S. Caux, Phys. Rev. Lett. 98, 150403 (2007); J. Stat. Mech. (2007) P08032.

[31] P. Le Doussal, P. Calabrese, A. Rosso in preparation.

[32] P.L. Ferrari and H. Spohn, J. Phys. A 38 L557 (2005).

[33] For convenience we exchanged the DP endpoints, using that $Z_{w}$ is real and $H_{n}$ hermitian
[1] M. Kardar, G. Parisi and Y.C. Zhang, Phys. Rev. Lett. 56, 889 (1986). 